Service social

\title{
Belgique : Les violences homophobes en débat
}

\section{David Paternotte}

Volume 59, numéro 1, 2013

L'homophobie et les tentatives de suicide et la résilience chez les jeunes LGBTQ

URI : https://id.erudit.org/iderudit/1017482ar

DOI : https://doi.org/10.7202/1017482ar

Aller au sommaire du numéro

\section{Éditeur(s)}

École de service social de l’Université Laval

ISSN

1708-1734 (numérique)

Découvrir la revue

Citer cet article

Paternotte, D. (2013). Belgique : Les violences homophobes en débat. Service social, 59(1), 104-107. https://doi.org/10.7202/1017482ar d'utilisation que vous pouvez consulter en ligne.

https://apropos.erudit.org/fr/usagers/politique-dutilisation/ 


\section{Belgique : Les violences homophobes en débat}

\section{David Paternotte}

Depuis 2011, un débat sur les violences sexistes et homophobes a vu le jour en Belgique. Celui-ci a débuté par la médiatisation d'agressions, particulièrement dans le centre de Bruxelles. Ainsi, fin 2011, un des principaux bars gays de la ville a été le théâtre d'un fait divers violent. Deux hommes éméchés sont entrés dans ce bar et ont commencé à insulter les personnes présentes. Si les détails de l'événement ne sont pas connus, un des employés a saisi un couteau pour se défendre et a poignardé les «agresseurs ». Le bar a été fermé pendant plusieurs semaines. En 2012, deux meurtres homophobes - les premiers recensés en Belgique - se sont produits à Liège. La première victime était un jeune gay musulman d'origine magrébine, violenté et poignardé avant que sa dépouille ne soit abandonnée dans les bois; la deuxième, un sexagénaire présent sur un lieu de drague et assassiné à coups de marteau.

Ces événements ont ébranlé les consciences et ont provoqué de nombreuses réactions. Ils ont aussi placé le thème des violences homophobes au centre des agendas politiques et militants. De nouveaux acteurs sont apparus, comme le groupe bruxellois Outrage !, qui est aussi à l'origine d'une application controversée pour smartphone permettant de cartographier les endroits réputés « dangereux » de la ville. Le premier ministre Elio Du Rupo, ouvertement homosexuel, a convoqué une réunion avec les différents acteurs de terrain afin de mettre sur pied un plan national de lutte contre l'homophobie. Une loi sur le sexisme a été annoncée et les peines pour délit d'homophobie seront durcies. La Ville de Bruxelles a enfin étendu la portée des sanctions administratives aux insultes, y compris sexistes et homophobes.

Cette polémique a rebondi au cours de l'automne 2012. Durant l'été, une étudiante en communication flamande, Sofie Peeters, a diffusé un documentaire intitulé « Femme de la rue » pour dénoncer la persistance du sexisme dans les rues de Bruxelles, en utilisant une caméra cachée pour montrer l'hostilité qu'elle rencontrait. Ce reportage diffusé par la télévision publique flamande a provoqué un grand émoi dans l'ensemble du pays. Fin octobre, un journaliste et militant flamand, Sven Pichal, a repris ce procédé pour dénoncer l'homophobie. II s'est baladé main dans la main avec un autre militant dans certains quartiers du centre de Bruxelles et d'Anvers. Ce reportage, intitulé « Hommes de la rue » en écho au travail de Sofie Peeters, a aussi été présenté par la télévision publique flamande et a été suivi le lendemain d'un sujet dans l'émission à grande audience Panorama. Ces deux reportages ont relancé la discussion et le sujet a occupé les devants de la presse flamande - et dans une moindre mesure francophone - pendant plusieurs jours. Le texte ci-dessous, présenté dans une version étendue, a été publié le 16 novembre 2012 en tant que tribune dans le quotidien La Libre Belgique. Il fait suite à une intervention plus courte de l'auteur dans l'édition du 8 novembre 2012 du journal Le Soir.

Dans ce texte, je souhaitais interroger les cadres dans lesquels prend place ce débat et inviter à se méfier des pentes glissantes où il peut nous entraîner. Depuis sa publication, un nouvel incident a occupé l'actualité bruxelloise. Fin novembre, un étudiant de l'école supérieure 
flamande Hogeschool Universiteit Brussel, travesti dans le cadre des rites de bizutage, s'est fait violer par deux mineurs d'âge au centre de Bruxelles. La direction de l'établissement a alors conseillé de ne plus se travestir lors des fêtes étudiantes dans le but de protéger les étudiants. Cette décision, qui fait fi de la diversité des genres, tend ainsi à confirmer l'idée selon laquelle le cœur de la capitale de l'Europe est dangereux pour les femmes et les homosexuels. Le travail politique suit quant à lui son cours. Une loi sur le sexisme a notamment été annoncée.

\section{" Penser les violences homophobes À BruXelles "}

Ces derniers jours, les médias ont abondamment parlé d'homophobie. C'est incontestable, il y a des problèmes et l'homophobie reste un enjeu dans notre société. Toutefois, au-delà d'un constat qui est loin d'être neuf, il est fondamental de réfléchir à la manière dont ce débat est posé afin de ne pas se laisser entraîner sur des terrains dangereux.

\section{Interroger nos perceptions}

Qui voyage un peu saura que Bruxelles n'est pas une ville dangereuse. Ce constat vaut tant pour les gays et les lesbiennes que pour l'ensemble de la population. Cela ne veut pas dire que des incidents violents ne surviennent jamais. Mais il est crucial de les saisir pour ce qu'ils sont réellement. Au risque, sinon, de générer un climat d'insécurité qui ne correspond pas à la réalité. Et d'attiser des discours sécuritaires profitant à des acteurs politiques dont nous ne partageons peut-être pas les opinions.

Ces représentations alimentent l'idée de désordre et de mauvaise gestion des grandes villes. Cette idée est ancienne, tout particulièrement pour Bruxelles, et a été assénée à maintes reprises en Flandre au cours des dernières années. Sans affirmer que certains partis auraient orchestré ces sorties médiatiques, ni même que les journalistes à l'origine de ces reportages seraient de connivence avec ces mouvements, ce constat aide à comprendre pourquoi, aujourd'hui, ces préoccupations rencontrent un tel écho, particulièrement au nord du pays ${ }^{1}$.

On entend aussi souvent que ces agressions seraient en augmentation et que les gays et les lesbiennes vivaient plus tranquilles auparavant. Il est difficile, en l'absence d'études sérieuses, de confirmer ou d'infirmer cette hypothèse. Toutefois, combien de couples homosexuels se donnaient la main dans la rue il y a dix ou quinze ans ? Cette visibilité accrue est récente. En outre, s'il y a encore de tels incidents, plus de personnes homosexuelles dénoncent des actes ou des propos qu'elles auraient supportés en silence auparavant et le système de recueil de plaintes s'est amélioré. Ces deux avancées font nécessairement augmenter le nombre d'incidents répertoriés.

1. NDLA: La Flandre - une région où les différents partis de droite et de centre-droit remportent aujourd'hui $80 \%$ des suffrages - a connu une montée des discours sécuritaires depuis le début des années 1990. Au cours des dernières années, la N-VA (Nieuw-Vlaams Alliantie, principal parti nationaliste et premier parti de Flandre) s'en est fait le héraut tout en dénonçant la mauvaise gestion de Bruxelles, une ville majoritairement francophone. 
En d'autres mots, il faut se méfier d'un âge d'or qui n'a probablement jamais existé. Comme l'écrivait Milan Kundera, « les nuages oranges du couchant éclairent toute chose du charme de la nostalgie, même la guillotine ». On assiste plutôt aujourd'hui au réveil d'un long rêve nourri des avancées législatives de la dernière décennie. Ni l'ouverture du mariage ni celle de l'adoption - et encore moins le choix d'un premier ministre ouvertement homosexuel - ne suffisent en effet à faire disparaître l'homophobie.

\section{Saisir la question dans sa globalité}

En 1981, le démographe néerlandais Maks Banens a proposé le concept d'homoaversion, qu'il distinguait de celui d'antihomosexualité ${ }^{2}$. Ce concept ancien et un peu oublié permet, à la différence de la notion fourre-tout d'homophobie, de comprendre plus en finesse ce que est en train de se passer. En effet, l'homoaversion, qui prend place au niveau des sentiments, est structurelle, tandis que l'antihomosexualité s'inscrit dans des actes et des paroles et surgit souvent en réaction à une visibilité accrue de l'homosexualité. Si on note une diminution de l'homoaversion (les homosexuel-le-s sont globalement mieux accepté-e-s), l'antihomosexualité pourrait être en augmentation ou devenir plus virulente. Les récentes agressions traduiraient alors non une diminution de l'acception de l'homosexualité mais, au contraire, la radicalisation, parfois violente, de personnes de plus en plus minoritaires dans notre société.

Par ailleurs, la compréhension de ces attaques requiert une vision plus large. II est clair que cette question touche les normes de genre et sexuelles en général. Mais nombre d'observateurs ont aussi souligné des intersections avec l'origine ethnique, la culture ou encore la religion. Toutefois, un aspect du problème demeure curieusement absent : la classe sociale. Or, tout le monde sait que les quartiers représentés dans ces reportages sont pauvres et ont été longtemps laissés à l'abandon, que leurs habitants, victimes de diverses discriminations, sont souvent abonnés au chômage et aux emplois précaires ${ }^{3}$. En face, on nous présente des gays et des lesbiennes de classe moyenne, voire supérieure, qui se sont souvent établis parfois - de manière ostentatoire - dans ces quartiers bon marché et ont alimenté une dynamique de gentrification. Dans un tel contexte, l'homophobie est peut-être aussi pour partie la manifestation d'une autre sorte de rejet.

\section{Absolus sexuels}

Pendant longtemps, l'homosexualité ne désignait que des désirs et des pratiques amoureuses ou sexuelles. Et, malgré la mondialisation, c'est ainsi qu'on la conçoit toujours dans plusieurs parties du monde. Toutefois, chez nous, ces pratiques se sont doublées au fil du temps d'une identité (être homosexuel).

2. Maks Banens, De Homo-aversie. Een analyse van de maatschappelijke onderdrukking van de homoseksualiteit, Groningen, Historische Uitgeverij Groningen, 1981. Pour une traduction partielle en français: [http://www.banens.fr/1981_1.php]

3. Jacinthe Mazzocchetti, « Sentiments d'injustice et théorie du complot. Représentations d'adolescents migrants et issus des migrations africaines (Maroc et Afrique subsaharienne) dans des quartiers précaires de Bruxelles », Brussels Studies, 63, en ligne: [http://www.brusselsstudies.be/medias/publications/BruS63FR.pdf] 
Les débats actuels relèvent d'une telle conception. L'homosexualité y est présentée comme un trait fondamental de l'individu et certains dénoncent les agressions comme des attaques contre ce qu'ils sont. II serait illusoire de vouloir se débarrasser de cette conception, qui a de plus alimenté les luttes récentes pour les droits mais une insistance trop forte sur la dimension identitaire de l'homosexualité, qui devient pour certains un absolu, transforme ce débat en une opposition entre les homosexuel-le-s et d'autres groupes sociaux

Ce regard tend à occulter la réalité des gays et lesbiennes issus de ces groupes, dont on a peu parlé au cours des derniers jours, alors qu'ils sont les premiers exposés. Les rares mentions qu'on en faisait ressemblaient à des attentions « bienveillantes » sans que la parole ne leur soit véritablement donnée.

Surtout, cette conception nous empêche de construire la vision globale tant nécessaire aujourd'hui. II serait en effet plus fécond de poser le débat comme une question de vivre ensemble. La ville n'est pas simplement une mosaïque de quartiers ou d'individus mais un ensemble d'interactions et un espace partagé. II est donc urgent de réfléchir au modèle urbain que nous souhaitons et à la manière dont nous voulons et nous pouvons vivre ensemble.

David Paternotte

Chargé de recherches du FNRS Université libre de Bruxelles (ULB) 